\title{
Nonlinear optimization for mixed attenuation polyenergetic image reconstruction
}

Hu, Yunyi; Nagy, James G.; Zhang, Jianjun; Andersen, Martin S.

\section{Published in:}

Inverse Problems

Link to article, DOI:

$10.1088 / 1361-6420 / a b 0131$

Publication date:

2019

Document Version

Peer reviewed version

Link back to DTU Orbit

Citation (APA):

Hu, Y., Nagy, J. G., Zhang, J., \& Andersen, M. S. (2019). Nonlinear optimization for mixed attenuation polyenergetic image reconstruction. Inverse Problems, 35(6), [064004 ]. https://doi.org/10.1088/1361$6420 / a b 0131$

\section{General rights}

Copyright and moral rights for the publications made accessible in the public portal are retained by the authors and/or other copyright owners and it is a condition of accessing publications that users recognise and abide by the legal requirements associated with these rights.

- Users may download and print one copy of any publication from the public portal for the purpose of private study or research.

- You may not further distribute the material or use it for any profit-making activity or commercial gain

- You may freely distribute the URL identifying the publication in the public portal

If you believe that this document breaches copyright please contact us providing details, and we will remove access to the work immediately and investigate your claim. 


\title{
Nonlinear Optimization for Mixed Attenuation Polyenergetic Image Reconstruction
}

\author{
Yunyi $\mathrm{Hu}^{1}$, James G. Nagy ${ }^{1}$, Jianjun Zhang ${ }^{2}$, and \\ Martin S. Andersen ${ }^{3}$ \\ ${ }^{1}$ Department of Mathematics, Emory University, Atlanta, GA 30322 USA \\ ${ }^{2}$ Department of Mathematics, Shanghai University, Shanghai, 200444, China \\ ${ }^{3}$ Department of Applied Mathematics and Computer Science, Technical University of \\ Denmark, 2800 Lyngby, Denmark \\ E-mail: yunyi.hu@emory.edu, jnagy@emory.edu, jjzhang@staff.shu.edu.cn, \\ mskan@dtu.dk
}

\begin{abstract}
With the emergence of new computed tomography (CT) machines, polyenergetic image reconstruction has become increasingly popular in recent years. By solving a nonlinear equation, we can obtain not only the visual structure of the object, but also quantitative information about the materials in the object. In this paper, we revisit the multi-material polyenergetic model and transform it into a nonlinear optimization problem that involves both equality and inequality constraints. In order to keep the second order derivative positive semi-definite, we propose a modified Hessian. In addition to the modified Hessian, a problem-specific nonlinear interior-point method is implemented to solve this problem. Moreover, total variation regularization is applied to stabilize the solutions. Both for full CT and limited angle cases, we can obtain images of high quality with this method. Numerical experiments illustrate the convergence, effectiveness, and significance of the proposed method.
\end{abstract}

Keywords: digital image reconstruction, interior-point method, beam hardening artifacts, modifed Hessian, multi-material polyenergetic model, total variation regularization 


\section{Introduction}

In imaging sciences, polyenergetic image reconstruction has gained tremendous popularity in the past few years. It is based on the physically meaningful assumption that x-rays are made up of a spectrum of energies rather than a single energy. In addition, if we consider the composition of the object as a mixture of a small number of known materials, the corresponding multi-material mathematical model can be written as

$$
\boldsymbol{b}=K(\boldsymbol{X}) \boldsymbol{s}+\boldsymbol{\eta},
$$

where $\boldsymbol{b}$ is a vector of projection data, $\boldsymbol{s}$ is a vector containing spectral information of the corresponding energies of the source x-ray, and $\boldsymbol{\eta}$ is the noise term. $K(\boldsymbol{X})=$ $\exp \left(-\boldsymbol{A} \boldsymbol{X} \boldsymbol{C}^{T}\right)$, where exponentiation is done element-wise, $\boldsymbol{A}$ is a matrix that is related to the quantitative information of the ray trace, $\boldsymbol{C}$ is a matrix of (known) material specific attenuation coefficients, and $\boldsymbol{X}$ is a matrix of unknowns, whose columns correspond to the weights of each of the known materials of the object being imaged. The forward operator is bounded, so it does not have a continuous inverse in infinitedimensional space, and the discrete problem inherits this property of ill-posedness. Therefore, computing an accurate approximation of $\boldsymbol{X}$ is a challenging problem.

In order to solve this problem, we could make the incorrect assumption that the x-ray beam is emitted at a single energy, and substitute the vector $s$ with a scalar approximation, such as an average value $\bar{s}$. Accordingly, the attenuation coefficients, $\boldsymbol{C}$, are chosen corresponding to the single energy $\bar{s}$. In this case, we can divide the scalar $\bar{s}$ on both sides of equation (1), apply a natural logarithm to the data and model, to obtain a linear system. This results in a significantly simplified model on which various well studied algorithms for linear ill-posed inverse problems can be used. However, there exist two problems. First, it is not fundamentally true that the x-ray is single-energy, which can cause so-called beam hardening artifacts $[1,2]$. Second, we cannot reconstruct the composition of materials of the object with a single set of monoenergetic measurements. Because of these problems, Elbakri and Fessler [3] and Chung et al. [4] suggest that we could use a 2-material model with a polyenergetic assumption on the source x-ray. Moreover, Mejia-Bustamante et al. [5] extended this idea, and provided a GPU implementation [6]. We remark that the problem can be reformulated in terms of mass attenuation coefficients (see, e.g., [3]), which encodes density information into the mathematical model. This can be important in cases when materials have the same chemical composition but different densities. However, it does not fundamentally change our proposed optimization approach, so in the remainder of the paper we use the model based on linear attenuation coefficients. To solve the nonlinear inverse problem in [5, 6], a maximum likelihood function was used to represent the evidence with respect to parameters $\boldsymbol{X}$. The gradient descent method is used to solve the resulting optimization problem, with an implicit enforcement of the constraint that in each voxel, the weights across all materials should sum to one. In this condition, the algorithm is easy to implement and we can reconstruct the weights corresponding to 
different materials. However, the numerical experiments are not in favor of this method. The Poisson likelihood function is nonlinear, is likely to have multiple local minimizers and the convergence speed might be slow. In addition, while it includes the equality constraints, it ignores bound constraints and does not explicitly enforce regularization, which limits its effectiveness.

From the physical point of view, the Poisson likelihood function tends to put more weight on higher measurements, whereas a least square loss function puts equal weights on all measurements [7]. In this paper, we use the Poisson likelihood function as loss function and total variation as regularization. Basically, we assume that

$$
\boldsymbol{b} \sim \text { Poisson }(K(\boldsymbol{X}) \boldsymbol{s})
$$

and build the negative log-likelihood function $f(\boldsymbol{X})$. The goal is to solve the regularized nonlinear optimization problem

$$
\min _{\boldsymbol{X} \in \boldsymbol{P}} f(\boldsymbol{X})+\alpha R(\boldsymbol{X}),
$$

where $R(\boldsymbol{X})$ is the regularization term and $\alpha$ is the regularization parameter. $\boldsymbol{P}=$ $\left\{\boldsymbol{X} \mid \boldsymbol{X} \mathbf{1}_{N_{m}}=\mathbf{1}_{N_{v}}, \mathbf{0} \leqslant \boldsymbol{X} \leqslant \mathbf{1}\right\}$ represents the set of constraints, and the variable $\boldsymbol{X}$ is both restricted by equality and inequality constraints. That is, we require that the entries of the weight matrix $\boldsymbol{X}$ are bounded below by zero and bounded above by one. We also impose that the summation of different materials in each voxel (pixel if 2D) should be one, which means that the row sum of matrix $\boldsymbol{X}$ is one. In general, the original problem has been transformed into a large-scale nonlinear optimization problem under equality and inequality constraints.

Solving this inverse problem is challenging. In addition to the ill-posedness that results from the objective function, we should be concerned about the equality and inequality constraints. In this paper, we use a problem-specific nonlinear interior-point method. Moreover, we provide a modified Hessian that is close to the true Hessian and is also positive semi-definite. The interior-point method has proven to be efficient and stable for solving nonlinear optimization problems, and with the implementation of our modified Hessian, we can simultaneously obtain an accurate approximation of the true Hessian and avoid negative curvature. Numerical experiments have shown very promising results for this method.

This paper is organized in the following way. In Section 2, we present the general multi-material polyenergetic model for computed tomography. In Section 3, we show how to derive the specific multi-material model from the general model. The discretization of this model is included in Section 3 as well. In Section 4, we revise this model to one that is more amenable to numerical implementation and formulate an optimization problem that is based on this model. The standard form of this optimization problem and the method to solve it are discussed in Section 5. Moreover, numerical experiments and comparison with existing method is presented in Section 6. In Section 7, we conclude with merits and limitations about the model and the optimization method. 


\section{General Model for Polyenergetic Computed Tomography}

In computed tomography, x-ray beams are emitted (usually in a cone) from a source at known energies and are directed to pass through an object under investigation, after which the remaining energy of the x-ray beams are measured at a detector. See Figure 1 for a 2D illustration. The amount of energy lost as the x-ray beams pass through the
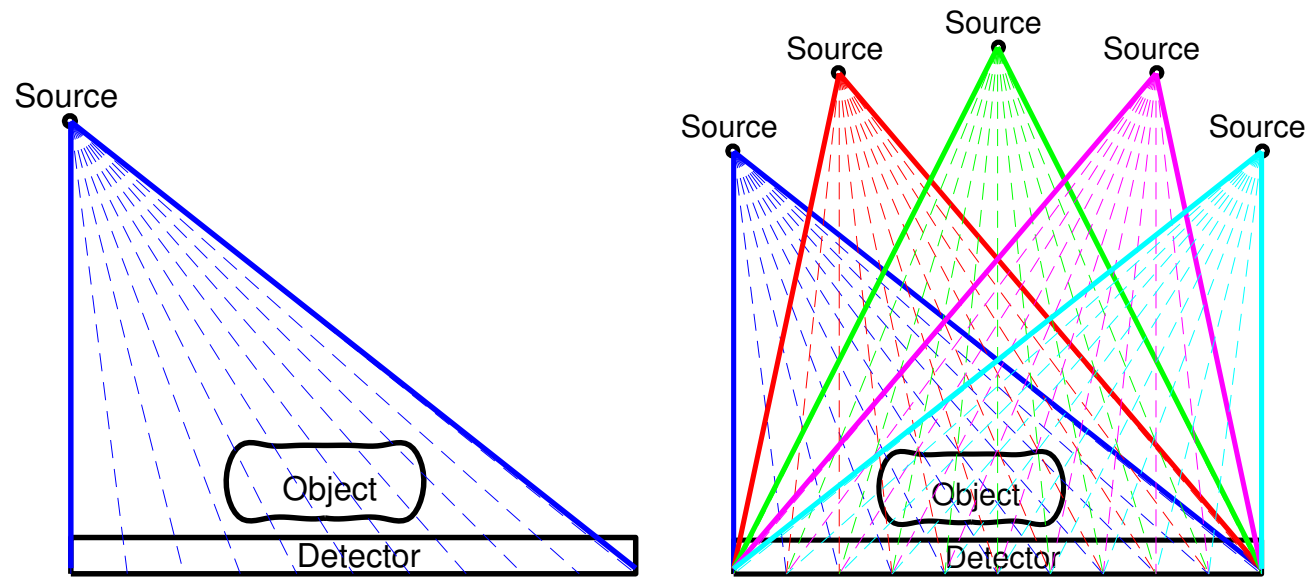

Figure 1. Illustration of a $2 \mathrm{D} \mathrm{CT}$ imaging setup. The left illustrates how x-ray beams are emitted from the source in a cone, pass through an object of interest, and are then measured at the detector. The right illustrates how the source might be rotated around the object to collect additional data. In this illustration we assume the detector remains stationary, which is often the case in limited angle tomography applications such as tomosynthesis, but it should be obvious how the illustration would be modified if the detector rotates with the source.

object is referred to as attenuation. The amount of attenuation depends on the energy of the x-ray beams, and on the material through which it penetrates; low dose energies are more easily attenuated, and denser materials have higher attenuation properties. The detector is typically partitioned into a grid of bins; in a full 3D model, these are often loosely referred to as detector pixels. If the $3 \mathrm{D}$ object is discretized into a grid of small volume elements (called voxels), then each voxel can be associated with a particular attenuation value, referred to as an attenuation coefficient. The problem of CT image reconstruction is to determine these attenuation coefficients from a sequence of measured projection data, which is obtained by rotating the source (at least partially) around the object; again, we refer to Figure 1 for a 2D illustration of the data collection process.

Using Beer's law [8], the energy integrating detector model can be written as

$$
\begin{aligned}
& b_{i}^{(\theta)}=\int_{E} S(e) \exp \left(-\int_{t \in \ell} \mu(\vec{x}(t), e) \mathrm{d} t\right) \mathrm{d} e+\eta_{i}^{(\theta)}, \\
& \left\{\begin{array}{l}
i=1,2, \cdots, N_{p}, \\
\theta=1,2, \cdots, N_{\theta},
\end{array}\right.
\end{aligned}
$$

where 
- $N_{p}$ is the number of pixels in the detector.

- $N_{\theta}$ is the number of projection angles.

- $S(e)$ represents the system spectral response, which is a product of x-ray energy with the number of incident photons at that energy.

- The outer integral is over all x-ray energies emitted from the source, and the inner integral is along lines that follow the x-ray beam paths through the object.

- $\mu(\vec{x}(t), e)$ denotes the attenuation coefficient, which is related to the position function $\vec{x}(t)$ and the energy level $e$.

- $\eta_{i}^{(\theta)}$ represents unknown errors in the measurements, which can include x-ray scatter and electronic noise.

If we discretize the model (4) over the a range of energies $E$ and along a ray traced by $\ell$, we obtain the discrete version of the previous equation as

$$
\begin{aligned}
& b_{i}^{(\theta)}=\sum_{e=1}^{N_{e}} s_{e} \exp \left(-\sum_{j=1}^{N_{v}} a_{i, j}^{(\theta)} \mu_{j, e}\right)+\eta_{i}^{(\theta)}, \\
& \left\{\begin{array}{l}
i=1,2, \cdots, N_{p}, \\
\theta=1,2, \cdots, N_{\theta},
\end{array}\right.
\end{aligned}
$$

where

- $a_{i, j}^{(\theta)}$ denotes the length of the x-ray beam that passes through voxel $j$ of the object and is incident onto $i$ th bin in the detector, with source at angle $\theta$.

- $\mu_{j, e}$ is the unknown variable that we want to reconstruct, which represents the attenuation coefficient for composite (mixture) materials, in voxel $j$ and at energy level $e$.

The traditional methods to solve this inverse problem are mostly based on filtered back-projection (FBP) [9]. If we assume the source to be monoenergetic and the source energy is $s_{e}$, then we can build a linear inverse problem by dividing $s_{e}$ on both sides and by applying the natural logarithm function to the data and to the model. Other approaches can be used to solve this linear inverse problem, such as incorporating different regularization schemes. These usually involve applying appropriate iterative optimization methods, such as the conjugate gradient method [10].

However, the images obtained from traditional methods on the simplified linear model might lead to significant beam hardening artifacts when the object is made up of several very distinct materials, such as bone and soft tissue. In addition, the linear models cannot be used to recognize the the actual types materials from the results, nor can they separate different materials when they are mixed [11]. Moreover, the traditional methods are unstable when it comes to the limited angle cases. For these reasons, we consider the full nonlinear polyenergetic model. 


\section{Multi-material Model for Polyenergetic Computed Tomography}

In reality, x-rays are composed of a spectrum of energies rather than a single energy. Meanwhile, the object is often made up of more than just one material and these materials are usually mixed in several areas of the object. Because of the restrictions of this basic model, a model that combines multiple materials with distinct energy fluences has been considered; see, for example, [4] and the references therein.

It is hard to solve $\mu_{j, e}$ directly from the equation (5) because it has a large amount of unknown variables. The subindex $j$ ranges from 1 to number of voxels and $e$ is within the scope of number of energies. To facilitate the process of finding solutions, we can think about how to reduce the size of unknown variables. To shrink the number of unknown variables, we can exploit material decomposition [11] by expressing each attenuation coefficient, $\mu_{j, e}$, as a sum of product of weights $w_{j, m}$ and known material specific attenuation coefficients, $u_{m, e}$; that is,

$$
\mu_{j, e}=\sum_{m=1}^{N_{m}} w_{j, m} u_{m, e} .
$$

By assumption, the attenuation coefficient for the $m$-th material and energy level $e$ is already known and the only unknown variable is weight $w_{j, m}$. By using this expression, we have transformed the goal of solving $\mu_{j, e}$ to the target of solving for $w_{j, m}$, which is dependent on the number of voxels and number of materials. Usually, the number of materials is 2 or 3 and it is significantly fewer than the number of energies. To simplify the problem, we also assume that the sum of weights inside each voxel is equivalent to 1. That is to say,

$$
\sum_{m=1}^{N_{m}} w_{j, m}=1 .
$$

In this paper, we limit the discussion to 2 to 3 materials, but note that for energy discriminating detectors, separating more materials is feasible. With equations (6) and (7), the equation (5) is approximately equivalent to

$$
b_{i}^{(\theta)}=\sum_{e=1}^{N_{e}} s_{e} \exp \left(-\sum_{j=1}^{N_{v}} \sum_{m=1}^{N_{m}} a_{i j}^{(\theta)} w_{j, m} u_{m, e}\right)+\eta_{i}^{(\theta)} .
$$


We can collect all variables in terms of vectors and matrices as

$$
\begin{gathered}
\boldsymbol{b}=\left[\begin{array}{c}
\boldsymbol{b}^{(1)} \\
\boldsymbol{b}^{(2)} \\
\vdots \\
\boldsymbol{b}^{(\theta)}
\end{array}\right], \quad \boldsymbol{A}=\left[\begin{array}{c}
\boldsymbol{A}^{(\mathbf{1})} \\
\boldsymbol{A}^{(\mathbf{2})} \\
\vdots \\
\boldsymbol{A}^{(\boldsymbol{\theta})}
\end{array}\right], \quad \boldsymbol{s}=\left[\begin{array}{c}
s_{1} \\
s_{2} \\
\vdots \\
s_{N_{e}}
\end{array}\right], \\
\boldsymbol{\eta}=\left[\begin{array}{c}
\eta^{(1)} \\
\eta^{(2)} \\
\vdots \\
\eta_{(\theta)}
\end{array}\right], \quad \boldsymbol{X}=\left[\begin{array}{cccc}
w_{1,1} & w_{1,2} & \cdots & w_{1, N_{m}} \\
w_{2,1} & w_{2,2} & \cdots & w_{2, N_{m}} \\
\vdots & \vdots & \ddots & \vdots \\
w_{N_{v}, 1} & w_{N_{v}, 2} & \cdots & w_{N_{v}, N_{m}}
\end{array}\right] \\
\text { and } \boldsymbol{C}=\left[\begin{array}{ccccc}
\mu_{1,1} & \mu_{1,2} & \cdots & \mu_{1, N_{m}} \\
\mu_{2,1} & \mu_{2,2} & \cdots & \mu_{2, N_{m}} \\
\vdots & \vdots & \ddots & \vdots \\
\mu_{N_{e}, 1} & \mu_{N_{e}, 2} & \cdots & \mu_{N_{e}, N_{m}}
\end{array}\right] .
\end{gathered}
$$

By using these notations, the discrete model (8) can be expressed as

$$
\boldsymbol{b}=\exp \left(-\boldsymbol{A} \boldsymbol{X} \boldsymbol{C}^{T}\right) \boldsymbol{s}+\boldsymbol{\eta} .
$$

We have so far obtained the standard form of the polyenergetic multi-material model. Based on equation (10), the goal is to solve for the unknown weight matrix $\boldsymbol{X}$ such that $\boldsymbol{X} \in \boldsymbol{P}$, where $\boldsymbol{P}=\left\{\boldsymbol{X} \mid \boldsymbol{X} \mathbf{1}_{N_{m}}=\mathbf{1}_{N_{v}}, \mathbf{0} \leqslant \boldsymbol{X} \leqslant \mathbf{1}\right\} . \mathbf{1}_{N_{m}}$ and $\mathbf{1}_{N_{v}}$ are vectors of ones of length $N_{m}$ and $N_{v}$, respectively.

With different energy levels, the forward model is nonlinear and it is not possible to transform it into an equivalent linear model. Mejia-Bustamante et al. [5] use the assumption that each entry in $\boldsymbol{b}$ follows a Poisson distribution,

$$
\boldsymbol{b} \sim \text { Poisson }\left(\exp \left(-\boldsymbol{A} \boldsymbol{X} \boldsymbol{C}^{T}\right) \boldsymbol{s}\right)
$$

With this assumption, one can formulate a maximum likelihood (MLE) function based on the Poisson distribution. Moreover, if the measurements strictly follow the Poisson distribution, then it is naturally included with this model. In [5], a negative log-

likelihood is used as the objective function and approximations of $\boldsymbol{X}$ are computed using a gradient descent method, with equality constraints on weights implicitly incorporated.

However, they do not provide a clear matrix-vector form for the optimization problem. In the next session, we will discuss how to use Kronecker product and its properties to derive the log-likelihood function as well as the gradient and modified Hessian. Moreover, we want to obtain faster convergence and robust reconstruction so we use a nonlinear interior point trust region method to solve it. It has superlinear convergence speed and is prone to large-scale problems.

\section{Mathematical Model Revisited}

In this section we revise the previous model so that it will be easier to include bound constraints and additional regularization operators. First note that if the weights 
(unknowns) are stored as a matrix, then differentiation results in tensors, requiring tedious bookkeeping in the computations. In this work we instead rewrite the function to put the unknowns in vector form, and differentiate the objective function with respect to this vector. Notice that

$$
\operatorname{vec}\left(\boldsymbol{A} \boldsymbol{X} \boldsymbol{C}^{T}\right)=(\boldsymbol{C} \otimes \boldsymbol{A}) \operatorname{vec}(\boldsymbol{X}),
$$

where $\operatorname{vec}(\cdot)$ reshapes a given matrix into a vector by stacking the columns on top of each other. Therefore, we can rewrite (10) as

$$
\boldsymbol{b}=\left(\boldsymbol{s}^{T} \otimes \boldsymbol{I}\right) \exp [-(\boldsymbol{C} \otimes \boldsymbol{A}) \operatorname{vec}(\boldsymbol{X})]+\boldsymbol{\eta} .
$$

If we let

$$
\boldsymbol{x}=\operatorname{vec}(\boldsymbol{X}) \quad \text { and } \quad K(\boldsymbol{x})=\exp \{-(\boldsymbol{C} \otimes \boldsymbol{A}) \boldsymbol{x}\},
$$

then equation (13) is equivalent to

$$
\boldsymbol{b}=\left(\boldsymbol{s}^{T} \otimes \boldsymbol{I}\right) K(\boldsymbol{x})+\boldsymbol{\eta} .
$$

In this problem, we assume that each element of measured data, $\boldsymbol{b}_{i}$, follows a Poisson distribution with mean $\left[\left(\boldsymbol{s}^{T} \otimes \boldsymbol{I}\right) K(\boldsymbol{x})\right]_{i}$. That is to say,

$$
b_{i} \sim \text { Poisson }\left(\left[\left(\boldsymbol{s}^{T} \otimes \boldsymbol{I}\right) K(\boldsymbol{x})\right]_{i}\right) .
$$

Based on this assumption, the corresponding probability density function can be expressed as

$$
f_{\boldsymbol{b}}(\boldsymbol{b} ; \boldsymbol{x})=\prod_{i=1}^{N_{p} \times N_{\theta}} \frac{\left[\left(\boldsymbol{s}^{T} \otimes \boldsymbol{I}\right) K(\boldsymbol{x})\right]_{i}^{b_{i}} \exp \left(\left[-\left(\boldsymbol{s}^{T} \otimes \boldsymbol{I}\right) K(\boldsymbol{x})\right]_{i}\right)}{b_{i} !} .
$$

If we ignore the constant term, the corresponding likelihood function is

$$
L(\boldsymbol{x} ; \boldsymbol{b})=\prod_{i=1}^{N_{p} \times N_{\theta}}\left[\left(\boldsymbol{s}^{T} \otimes \boldsymbol{I}\right) K(\boldsymbol{x})\right]_{i}^{b_{i}} \exp \left(\left[-\left(\boldsymbol{s}^{T} \otimes \boldsymbol{I}\right) K(\boldsymbol{x})\right]_{i}\right) .
$$

The log-likelihood function can be represented as

$$
l(\boldsymbol{x} ; \boldsymbol{b})=\sum_{i=1}^{N_{p} \times N_{\theta}}\left\{b_{i} \log \left[\left(\boldsymbol{s}^{T} \otimes \boldsymbol{I}\right) K(\boldsymbol{x})\right]_{i}-\left[\left(\boldsymbol{s}^{T} \otimes \boldsymbol{I}\right) K(\boldsymbol{x})\right]_{i}\right\} .
$$

To maximize the log-likelihood function, it is equivalent to minimizing the negative log-likelihood function. So the objective function is

$$
\begin{aligned}
l(\boldsymbol{x} ; \boldsymbol{b}) & =\sum_{i=1}^{N_{p} \times N_{\theta}}\left\{\left[\left(\boldsymbol{s}^{T} \otimes \boldsymbol{I}\right) K(\boldsymbol{x})\right]_{i}-b_{i} \log \left[\left(\boldsymbol{s}^{T} \otimes \boldsymbol{I}\right) K(\boldsymbol{x})\right]_{i}\right\} . \\
& =\mathbf{1}_{N_{p} \times N_{\theta}}^{T}\left(\boldsymbol{s}^{T} \otimes \boldsymbol{I}\right) K(\boldsymbol{x})-\boldsymbol{b}^{T} \log \left[\left(\boldsymbol{s}^{T} \otimes \boldsymbol{I}\right) K(\boldsymbol{x})\right],
\end{aligned}
$$


where $\mathbf{1}_{N_{p} \times N_{\theta}}$ is a vector of all ones of length $N_{p} \times N_{\theta}$. Meanwhile, both the bound constraint and the constraint (7) should be included. The bound constraint is equivalent to $\mathbf{0} \leqslant \boldsymbol{x} \leqslant \mathbf{1}$ and we can rewrite the constraint (7) in a matrix-vector form as

$$
\boldsymbol{A}_{e q} \boldsymbol{x}=\mathbf{1}_{N_{v}}
$$

where $\boldsymbol{A}_{e q}$ is a matrix of the form

$$
\boldsymbol{A}_{e q}=\mathbf{1}_{N_{m}}^{T} \otimes \boldsymbol{I}_{N_{v}}
$$

$\mathbf{1}_{N_{m}}$ is a vector of ones of length $N_{m}$. $\boldsymbol{I}_{N_{v}}$ is an identity matrix of size $N_{v} \times N_{v}$. In the following sections, we use $\boldsymbol{I}$ to represent the identity matrix if the size of this matrix is clear to identify.

With the objective function (20), we can construct an optimization problem by combining the regularization term and the constraints:

$$
\begin{array}{cl}
\min _{\mathbf{0} \leqslant \boldsymbol{x} \leqslant \mathbf{1}} & f(\boldsymbol{x})+R(\boldsymbol{x}) \\
\text { subject to } & \boldsymbol{A}_{e q} \boldsymbol{x}=\mathbf{1}_{N_{v}}, \\
& \boldsymbol{A}_{e q}=\mathbf{1}_{N_{m}}^{T} \otimes \boldsymbol{I}_{N_{v}} .
\end{array}
$$

In the problem $(23), f(\boldsymbol{x})=l(\boldsymbol{x} ; \boldsymbol{b})$ and $R(\boldsymbol{x})$ represents the regularization term, which is used to penalize the variable $\boldsymbol{x}$. For this problem, we choose total variation regularization to preserve the edges. Using these notations, we can express the set of constraints as $\boldsymbol{Q}=\left\{\boldsymbol{x} \mid \boldsymbol{A}_{e q} \boldsymbol{x}=\mathbf{1}_{N_{v}}, \mathbf{0} \leqslant \boldsymbol{x} \leqslant \mathbf{1}\right\}$. So the notation can be simplified as

$$
\min _{\boldsymbol{x} \in \boldsymbol{Q}} f(\boldsymbol{x})+R(\boldsymbol{x}) .
$$

We let $\boldsymbol{x}_{k}$ be the $k$-th column in matrix $\boldsymbol{X}$ and $\boldsymbol{X}_{k}$ be the corresponding reshaped image of $\boldsymbol{x}_{k}$. The total variation regularization for the $k$-th material can be represented as [12]:

$$
R\left(\boldsymbol{X}_{k}\right)=\sum_{i=1}^{n} \sum_{j=1}^{n}\left(\left(\boldsymbol{X}_{k} \boldsymbol{D}^{T}\right)_{i j}^{2}+\left(\boldsymbol{D} \boldsymbol{X}_{k}\right)_{i j}^{2}\right)^{1 / 2}
$$

where $\boldsymbol{D}$ is either a forward, backward or central first order finite difference matrix and $n$ is the resolution. For all $m$ materials, the regularization term can be expressed as

$$
R(\boldsymbol{X})=\sum_{k=1}^{m} \frac{\alpha_{k}}{2} R\left(\boldsymbol{X}_{k}\right)
$$

where $\alpha_{k}$ is the regularization parameter. Other forms of regularization, such as the discrete Laplacian, can also be used with this framework. For simplicity, we use zero boundary conditions when we construct the matrix $\boldsymbol{D}$. The gradient to the objective function can be expressed as

$$
\nabla l(\boldsymbol{x})=-\left(\boldsymbol{C}^{T} \otimes \boldsymbol{A}^{T}\right) \operatorname{diag}\{K(\boldsymbol{x})\}(\boldsymbol{s} \otimes \boldsymbol{I})\left\{\mathbf{1}_{N_{p} \times N_{\theta}}-\boldsymbol{b} \oslash\left[\left(\boldsymbol{s}^{T} \otimes \boldsymbol{I}\right) K(\boldsymbol{x})\right]\right\} .
$$


Differentiating the gradient with respect to $\boldsymbol{x}$, the Hessian can be represented as a summation of two parts:

$$
\nabla^{2} l(\boldsymbol{x})=H_{1}(\boldsymbol{x})+H_{2}(\boldsymbol{x})
$$

where

$$
\begin{aligned}
H_{1}(\boldsymbol{x})= & \left(\boldsymbol{C}^{T} \otimes \boldsymbol{A}^{T}\right) \operatorname{diag}\{K(\boldsymbol{x})\} \operatorname{diag}\left\{(\boldsymbol{s} \otimes \boldsymbol{I})\left(\mathbf{1}_{N_{p} \times N_{\theta}}-\boldsymbol{b} \oslash\left[\left(\boldsymbol{s}^{T} \otimes \boldsymbol{I}\right) K(\boldsymbol{x})\right]\right)\right\}(\boldsymbol{C} \otimes \boldsymbol{A}) \\
H_{2}(\boldsymbol{x})= & \left(\boldsymbol{C}^{T} \otimes \boldsymbol{A}^{T}\right) \operatorname{diag}\{K(\boldsymbol{x})\}(\boldsymbol{s} \otimes \boldsymbol{I}) \\
& \operatorname{diag}\left\{\boldsymbol{b} \oslash\left[\left(\boldsymbol{S}^{T} \otimes \boldsymbol{I}\right) K(\boldsymbol{x})\right] \cdot{ }^{2}\right\}\left(\boldsymbol{s}^{T} \otimes \boldsymbol{I}\right) \operatorname{diag}\{K(\boldsymbol{x})\}(\boldsymbol{C} \otimes \boldsymbol{A}) .
\end{aligned}
$$

From the previous expressions, we can see that $H_{2}(\boldsymbol{x})$ is the Gauss-Newton approximation to the true Hessian and it is always positive semidefinite. Even if $H_{1}(\boldsymbol{x})$ is indefinite, we can transform it into a positive semi-definite matrix by setting a threshold. Specifically, let $T(\boldsymbol{x})$ be defined as:

$$
T(\boldsymbol{x})=\max \left[\mathbf{0},(\boldsymbol{s} \otimes \boldsymbol{I})\left(\mathbf{1}_{N_{p} \times N_{\theta}}-\boldsymbol{b} \oslash\left[\left(\boldsymbol{s}^{T} \otimes \boldsymbol{I}\right) K(\boldsymbol{x})\right]\right)\right] .
$$

Then the new $\hat{H}_{1}(\boldsymbol{x})$ can be represented as

$$
\hat{H}_{1}(\boldsymbol{x})=\left(\boldsymbol{C}^{T} \otimes \boldsymbol{A}^{T}\right) \operatorname{diag}\{K(\boldsymbol{x})\} \operatorname{diag}\{T(\boldsymbol{x})\}(\boldsymbol{C} \otimes \boldsymbol{A}) .
$$

Moreover, we define the modified Hessian as

$$
\hat{H}(\boldsymbol{x})=\hat{H}_{1}(\boldsymbol{x})+H_{2}(\boldsymbol{x}) .
$$

With the modified Hessian, we can include most information about the true Hessian as well as keep it positive semi-definite. However, direct implementation of Newton's

method is not effective. To guarantee the feasibility of each step, we need to project the current step onto the boundaries. The projected solution might be far away from the desired solution and hard to improve later. Furthermore, it is difficult to maintain the equality constraint in each step without changing the original model.

\section{Implementation of Nonlinear Interior-point Method}

Recall that the optimization problem can be expressed as

$$
\begin{array}{rl}
\min _{\mathbf{0} \leqslant \boldsymbol{x} \leqslant \mathbf{1}} & f(\boldsymbol{x})+\alpha R(\boldsymbol{x}) \\
\text { subject to } & \boldsymbol{A}_{e q} \boldsymbol{x}=\mathbf{1},
\end{array}
$$

where $f(\boldsymbol{x})=l(\boldsymbol{x} ; \boldsymbol{b})$ and $R(\boldsymbol{x})$ is the regularization term.

To solve this constrained optimization problem, we use a nonlinear interior-point trust region method, which combines sequential quadratic programming, a trust region 
dogleg method, and a projected conjugate gradient algorithm [13, 14]. To apply this method, we firstly establish a barrier problem based on (33):

$$
\begin{array}{cl}
\min _{\boldsymbol{x}, \boldsymbol{z}} & f(\boldsymbol{x})+\alpha R(\boldsymbol{x})-\mu \sum_{i=1}^{2 N_{v}} \ln \left(z_{i}\right) \\
\text { subject to } & \boldsymbol{A}_{e q} \boldsymbol{x}-\mathbf{1}=\mathbf{0}, \\
& \boldsymbol{A}_{i e q} \boldsymbol{x}+\boldsymbol{b}_{i e q}+\boldsymbol{z}=\mathbf{0},
\end{array}
$$

where

$$
\boldsymbol{A}_{i e q}=\left[\begin{array}{c}
-\boldsymbol{I} \\
\boldsymbol{I}
\end{array}\right] \text { and } \boldsymbol{b}_{i e q}=\left[\begin{array}{c}
-\mathbf{1} \\
\mathbf{0}
\end{array}\right] .
$$

$\mu$ is the barrier parameter that should decrease to 0 and $\boldsymbol{z}$ is the vector of slack variables that ensure all entries remain positive. The permuted KKT condition corresponding to (34) can be written as

$$
\begin{aligned}
\nabla f(\boldsymbol{x})+\alpha \nabla R(\boldsymbol{x})+\boldsymbol{A}_{e q}^{T} \boldsymbol{\lambda}_{e q}+\boldsymbol{A}_{i e q}^{T} \boldsymbol{\lambda}_{i e q} & =\mathbf{0}, \\
\boldsymbol{Z} \boldsymbol{\lambda}_{i e q}-\mu \mathbf{1} & =\mathbf{0}, \\
\boldsymbol{A}_{e q} \boldsymbol{x}-\mathbf{1} & =\mathbf{0}, \\
\boldsymbol{A}_{i e q} \boldsymbol{x}+\boldsymbol{b}_{i e q}+\boldsymbol{z} & =\mathbf{0},
\end{aligned}
$$

where $\boldsymbol{\lambda}_{e q}$ and $\boldsymbol{\lambda}_{i e q}$ are the Lagrange multipliers corresponding to the equality and the inequality constraints, respectively. Furthermore, $\boldsymbol{\lambda}_{i e q}$ should be nonnegative. $\boldsymbol{Z}$ is a diagonal matrix and $\boldsymbol{Z}=\operatorname{diag}\left\{z_{1}, z_{2}, \cdots, z_{2 N_{e}}\right\}$. Compared with the original KKT system, this permuted KKT system is preferred because the matrix $\boldsymbol{Z}$ is bounded when the entries of $\boldsymbol{z}$ are approaching 0 . We can construct an error function based on this system:

$$
\begin{aligned}
E\left(\boldsymbol{x}, \boldsymbol{z}, \boldsymbol{\lambda}_{e q}, \boldsymbol{\lambda}_{i e q} ; \mu\right)=\max \{ & \| f(\boldsymbol{x})+\alpha \nabla R(\boldsymbol{x}) \\
& +\boldsymbol{A}_{e q}^{T} \boldsymbol{\lambda}_{e q}+\boldsymbol{A}_{i e q}^{T} \boldsymbol{\lambda}_{i e q} \|_{\infty}, \\
& \left\|\boldsymbol{Z} \lambda_{i e q}-\mu\right\|_{\infty},\left\|\boldsymbol{A}_{e q} \boldsymbol{x}-\mathbf{1}\right\|_{\infty}, \\
& \left.\left\|\boldsymbol{A}_{i e q} \boldsymbol{x}+\boldsymbol{b}_{i e q}+\boldsymbol{z}\right\|_{\infty}\right\} .
\end{aligned}
$$

If the error function is less than a tolerance, for example, $10^{-8}$, then we assume that we have solved this system approximately. The Newton system corresponding to the permuted KKT system can be written as

$$
D(\boldsymbol{x}) \boldsymbol{p}=-g(\boldsymbol{x})
$$


where

$$
\begin{aligned}
D(\boldsymbol{x})= & {\left[\begin{array}{cccc}
\hat{H}(\boldsymbol{x})+\alpha \nabla^{2} R(\boldsymbol{x}) & \mathbf{0} & \boldsymbol{A}_{e q}^{T} & \boldsymbol{A}_{i e q}^{T} \\
\mathbf{0} & \boldsymbol{\Lambda}_{i e q} & \mathbf{0} & \boldsymbol{Z} \\
\boldsymbol{A}_{e q} & \mathbf{0} & \mathbf{0} & \mathbf{0} \\
\boldsymbol{A}_{i e q} & \boldsymbol{I} & \mathbf{0} & \mathbf{0}
\end{array}\right], } \\
g(\boldsymbol{x})= & {\left[\begin{array}{c}
\nabla f(\boldsymbol{x})+\alpha \nabla R(\boldsymbol{x})+\boldsymbol{A}_{e q}^{T} \boldsymbol{\lambda}_{e q}+\boldsymbol{A}_{i e q}^{T} \boldsymbol{\lambda}_{i e q} \\
\boldsymbol{Z} \boldsymbol{\lambda}_{i e q}-\mu \mathbf{1} \\
\boldsymbol{A}_{e q} \boldsymbol{x}-\mathbf{1} \\
\boldsymbol{A}_{i e q} \boldsymbol{x}+\boldsymbol{b}_{i e q}+\boldsymbol{z}
\end{array}\right] } \\
\text { and } \boldsymbol{p} & =\left[\begin{array}{r}
\boldsymbol{p}_{x} \\
\boldsymbol{p}_{z} \\
\Delta \boldsymbol{\lambda}_{e q} \\
\Delta \boldsymbol{\lambda}_{i e q}
\end{array}\right] .
\end{aligned}
$$

In the matrix $D(\boldsymbol{x}), \boldsymbol{\Lambda}_{i e q}$ is a diagonal matrix with $\boldsymbol{\lambda}_{i e q}$ in the diagonal: $\boldsymbol{\Lambda}_{i e q}=$ $\operatorname{diag}\left\{\boldsymbol{\lambda}_{i e q}\right\}$. However, both direct and iterative methods to solve this system are computationally expensive, especially for large-scale problems. We therefore follow the strategy of Byrd et al.[15] by transforming the original problem into a sequential quadratic programming problem and solve by separating it into two subproblems. The first subproblem is called the normal subproblem, which can be solved by trustregion dogleg method, while the second subproblem, the tangential subproblem, can be solved by the projected conjugate gradient method. By applying the idea of sequential quadratic programming, we can construct an optimization problem as

$$
\begin{aligned}
& \min _{\boldsymbol{p}_{x}, \boldsymbol{p}_{z}} \nabla[f(\boldsymbol{x})+\alpha R(\boldsymbol{x})]^{T} \boldsymbol{p}_{x}+\frac{1}{2} \boldsymbol{p}_{x}^{T}\left[\hat{H}(\boldsymbol{x})+\alpha \nabla^{2} R(\boldsymbol{x})\right] \boldsymbol{p}_{x} \\
& -\mu \mathbf{1}^{T} \boldsymbol{Z}^{-1} \boldsymbol{p}_{z}+\frac{1}{2} \boldsymbol{p}_{z}^{T} \boldsymbol{\Sigma} \boldsymbol{p}_{z} \\
& \text { subject to } \boldsymbol{A}_{e q} \boldsymbol{p}_{x}+\boldsymbol{A}_{e q} \boldsymbol{x}-\mathbf{1}=\boldsymbol{r}_{e q} \text {, } \\
& \boldsymbol{A}_{i e q} \boldsymbol{p}_{x}+\boldsymbol{p}_{z}+\boldsymbol{A}_{i e q} \boldsymbol{x}+\boldsymbol{b}_{i e q}+\boldsymbol{z}=\boldsymbol{r}_{i e q}, \\
& \left\|\left[\begin{array}{ll}
\boldsymbol{p}_{x}^{T} & \boldsymbol{p}_{z}^{T} \boldsymbol{Z}^{-1}
\end{array}\right]\right\|_{2} \leqslant \Delta \\
& \boldsymbol{p}_{z} \geqslant-\tau \boldsymbol{z},
\end{aligned}
$$

where $\boldsymbol{\Sigma}=\boldsymbol{Z}^{-1} \boldsymbol{\Lambda}_{i e q}$. For this primal-dual system, we regard $\boldsymbol{\Sigma}=\boldsymbol{Z}^{-1} \boldsymbol{\Lambda}_{i e q}$ as an approximation to the second order derivative $\mu \boldsymbol{Z}^{-2} \cdot \boldsymbol{r}_{e q}$ and $\boldsymbol{r}_{i e q}$ are auxiliary variables to the linearized constraints. Moreover, the trust region constraint is used to guarantee a sufficient reduction in each step and stop the loop after a fixed number of iterations. The scale term $\boldsymbol{Z}^{-1}$ in the trust region constraint is used to keep slack variables away from zero without enough iterations. Meanwhile, the bound constraint is applied to guarantee the positivity of slack variables $\boldsymbol{z}$. That is to say, if the step is accepted, then we should have

$$
\boldsymbol{z}+\boldsymbol{p}_{z} \geqslant \boldsymbol{z}-\tau \boldsymbol{z}=(1-\tau) \boldsymbol{z}>0
$$


$\tau$ is a constant that is close to 1 , for example, $\tau=0.98$. If we let $\tilde{\boldsymbol{p}}_{z}=\boldsymbol{Z}^{-1} \boldsymbol{p}_{z}$, then we can rewrite (40) as

$$
\begin{aligned}
& \min _{\boldsymbol{p}_{x}, \tilde{\boldsymbol{p}}_{z}} \nabla[f(\boldsymbol{x})+\alpha R(\boldsymbol{x})]^{T} \boldsymbol{p}_{x}+\frac{1}{2} \boldsymbol{p}_{x}^{T}\left[\hat{H}(\boldsymbol{x})+\alpha \nabla^{2} R(\boldsymbol{x})\right] \boldsymbol{p}_{x} \\
& -\mu \mathbf{1}^{T} \tilde{\boldsymbol{p}}_{z}+\frac{1}{2} \tilde{\boldsymbol{p}}_{z}^{T} \boldsymbol{Z} \boldsymbol{\Sigma} \boldsymbol{Z} \tilde{\boldsymbol{p}}_{z} \\
& \text { subject to } \boldsymbol{A}_{e q} \boldsymbol{p}_{x}+\boldsymbol{A}_{e q} \boldsymbol{x}-\mathbf{1}=\boldsymbol{r}_{e q} \text {, } \\
& \boldsymbol{A}_{i e q} \boldsymbol{p}_{x}+\boldsymbol{Z} \tilde{\boldsymbol{p}}_{z}+\boldsymbol{A}_{i e q} \boldsymbol{x}+\boldsymbol{b}_{i e q}+\boldsymbol{z}=\boldsymbol{r}_{i e q}, \\
& \left\|\left[\begin{array}{ll}
\boldsymbol{p}_{x}^{T} & \tilde{\boldsymbol{p}}_{z}^{T}
\end{array}\right]\right\|_{2} \leqslant \Delta \text {, } \\
& \tilde{\boldsymbol{p}}_{z} \geqslant-\tau \text {. }
\end{aligned}
$$

With the help of anxiliary variables, we can separate (42) into two subproblems, the normal subproblem and the tangential subproblem. The normal subproblem can be expressed as

$$
\begin{aligned}
& \min _{\boldsymbol{v}_{x}, \boldsymbol{v}_{z}}\left\|\boldsymbol{A}_{e q} \boldsymbol{v}_{x}+\boldsymbol{A}_{e q} \boldsymbol{x}-\mathbf{1}\right\|_{2}^{2} \\
& +\left\|\boldsymbol{A}_{i e q} \boldsymbol{v}_{x}+\boldsymbol{Z} \boldsymbol{v}_{z}+\boldsymbol{A}_{i e q} \boldsymbol{x}+\boldsymbol{b}_{i e q}+\boldsymbol{z}\right\|_{2}^{2} \\
& \text { subject to }\left\|\left[\boldsymbol{v}_{x}^{T} \quad \boldsymbol{v}_{z}^{T}\right]\right\|_{2} \leqslant \zeta \Delta, \\
& \qquad \boldsymbol{v}_{z} \geqslant-\frac{\tau}{2},
\end{aligned}
$$

where $\zeta$ is a constant and $0<\zeta<1$, for exmaple, $\zeta=0.8$. Without the bound constraint, the normal subproblem is a standard form trust region problem, which can be solved by the trust region dogleg method. So we solve it by ignoring the bound constraint and test if the solution satisfies this constraint. If not, we conduct backtracking to maintain feasibility [16]. After solving the normal subproblem approximately, we obtain the residuals as

$$
\begin{aligned}
& \boldsymbol{r}_{e q}=\boldsymbol{A}_{e q} \boldsymbol{v}_{x}+\boldsymbol{A}_{e q} \boldsymbol{x}-\mathbf{1} \\
& \boldsymbol{r}_{i e q}=\boldsymbol{A}_{i e q} \boldsymbol{v}_{x}+\boldsymbol{Z} \boldsymbol{v}_{z}+\boldsymbol{A}_{i e q} \boldsymbol{x}+\boldsymbol{b}_{i e q}+\boldsymbol{z}
\end{aligned}
$$

By substituting the residuals for the same terms in (42), the original optimization problem can be represented as

$$
\begin{aligned}
& \min _{\boldsymbol{p}_{x}, \tilde{\boldsymbol{p}}_{z}} \nabla[f(\boldsymbol{x})+\alpha R(\boldsymbol{x})]^{T} \boldsymbol{p}_{x}+\frac{1}{2} \boldsymbol{p}_{x}^{T}\left[\hat{H}(\boldsymbol{x})+\alpha \nabla^{2} R(\boldsymbol{x})\right] \boldsymbol{p}_{x} \\
& -\mu \mathbf{1}^{T} \tilde{\boldsymbol{p}}_{z}+\frac{1}{2} \tilde{\boldsymbol{p}}_{z}^{T} \boldsymbol{Z} \boldsymbol{\Sigma} \boldsymbol{Z} \tilde{\boldsymbol{p}}_{z} \\
& \text { subject to } \boldsymbol{A}_{e q}\left(\boldsymbol{p}_{x}-\boldsymbol{v}_{x}\right)=0 \text {, } \\
& \boldsymbol{A}_{i e q}\left(\boldsymbol{p}_{x}-\boldsymbol{v}_{x}\right)+\boldsymbol{Z}\left(\tilde{\boldsymbol{p}}_{z}-\boldsymbol{v}_{z}\right)=0, \\
& \left\|\left[\begin{array}{ll}
\boldsymbol{p}_{x}^{T} & \tilde{\boldsymbol{p}}_{z}^{T}
\end{array}\right]\right\|_{2} \leqslant \Delta, \\
& \tilde{\boldsymbol{p}}_{z} \geqslant-\tau \text {. }
\end{aligned}
$$

If we ignore the last two constraints, this optimization problem is a standard form quadratic programming problem under linear equality constraints, which can be solved 
by the projected conjugate gradient method. In this situation, we ignore the bound constraint at first and stop the iteration when the desired tolerance is attained or the current step crosses the trust region. If the solution does not satisfy the bound constraint, then we backtrack and choose the last feasible step as the solution.

After we have obtained $\boldsymbol{p}_{x}$ and $\boldsymbol{p}_{z}$, we need to decide if we should accept them and update the current step as well as the size of the trust region. To realize this idea, we can construct a merit function based on the objective function and constraints from the original barrier problem to decide the actual reduction. For example, a merit function can be expressed as

$$
\begin{aligned}
\phi_{\nu}(\boldsymbol{x}, \boldsymbol{z})= & f(\boldsymbol{x})+\alpha R(\boldsymbol{x})-\mu \sum_{i=1}^{2 N_{v}} \ln \left(z_{i}\right)+\nu\left\|\boldsymbol{A}_{e q} \boldsymbol{x}-\mathbf{1}\right\|_{2} \\
& +\nu\left\|\boldsymbol{A}_{i e q} \boldsymbol{x}+\boldsymbol{b}_{i e q}+\boldsymbol{z}\right\|_{2}
\end{aligned}
$$

where $\nu>0$ is a penalty parameter. The actual reduction can be expressed as

$$
\operatorname{ared}(\boldsymbol{p})=\phi_{\nu}(\boldsymbol{x}, \boldsymbol{z})-\phi_{\nu}\left(\boldsymbol{x}+\boldsymbol{p}_{x}, \boldsymbol{z}+\boldsymbol{p}_{z}\right) \text {. }
$$

The predicted reduction can be constructed in many ways, most of which are based on the SQP problem and its constraints. For example, we can set up a function as

$$
\begin{aligned}
q_{\nu}(\boldsymbol{p})= & \nabla[f(\boldsymbol{x})+\alpha R(\boldsymbol{x})]^{T} \boldsymbol{p}_{x}+\frac{1}{2} \boldsymbol{p}_{x}^{T}[\hat{H}(\boldsymbol{x})+\alpha R(\boldsymbol{x})] \boldsymbol{p}_{x} \\
& -\mu \mathbf{1}^{T} \boldsymbol{Z}^{-1} \boldsymbol{p}_{z}+\frac{1}{2} \boldsymbol{p}_{z}^{T} \boldsymbol{\Sigma} \boldsymbol{p}_{z} \\
& +\left\|\left[\begin{array}{c}
\boldsymbol{A}_{e q} \boldsymbol{p}_{x}+\boldsymbol{A}_{e q} \boldsymbol{x}-\mathbf{1} \\
\boldsymbol{A}_{i e q} \boldsymbol{p}_{x}+\boldsymbol{p}_{z}+\boldsymbol{A}_{i e q} \boldsymbol{x}+\boldsymbol{b}_{i e q}+\boldsymbol{z}
\end{array}\right]\right\|_{2} .
\end{aligned}
$$

For $q_{\nu}(\boldsymbol{p})$, the predicted reduction is the difference between not taking any step and taking the obtained step $\boldsymbol{p}$, which can be indicated as

$$
\operatorname{pred}(\boldsymbol{p})=q_{\nu}(\mathbf{0})-q_{\nu}(\boldsymbol{p}),
$$

where the variable $\boldsymbol{p}$ is a concatenation of $\boldsymbol{p}_{x}$ and $\boldsymbol{p}_{z}$. For a tiny constant $\eta=10^{-8}$, if $\operatorname{ared}(\boldsymbol{p}) \geqslant \eta \operatorname{pred}(\boldsymbol{p})$, we accept $\boldsymbol{p}$ and update the current step. We will also update the trust region with a standard criteria based on the ratio ared $(\boldsymbol{p}) / \operatorname{pred}(\boldsymbol{p})$.

In conclusion, we implement this problem-specific nonlinear interior-point method to solve the corresponding optimization problem (34). (34) is a nonlinear optimization problem under linear and bound constraints. The objective function contains a nonlinear log-likelihood term and a regularization term. For the log-likelihood term, we calculate the gradient and modified Hessian as (27) and (32). For the regularization term, the total variation regularization is chosen to stabilize the solution. The modified Hessian is close to the true Hessian and it is positive semidefinite so solutions to the argumented Newton system are robust. Furthermore, the problem is prone to large-scale application since it is unnecessary to save the modified Hessian. We only need Hessian-vector multiplication 
when we implement Newton-CG method to solve the argumented Newton system. The cost of memory in each conjugate gradient iteration is close to an iteration of gradient descent. Therefore, nonlinear interior point trust region method is a perfect match to this problem.

\section{Numerical Experiments}

To test the model and algorithm, we generate a 2D image of size 128 by 128 and assume that the object is made up of three simulated materials that arise in polyenergetic image reconstruction - adipose, air and bones. For bones, we use the main component, calcium, to represent it. One application of polyenergetic image reconstruction is breast imaging, which requires low dose radiation for patients. To realize this application, we generate an energy spectrum with potential $26 \mathrm{KeV}$ with the help of function 'spektrSpectrum' [17]. We also select a low radiation dose of $1 \mathrm{e} 5$ total photons for the x-ray energy spectrum. The corresponding spectrum is shown in Figure (2). From Figure (2), we can find that the photon flux density is above zero when the energy is between $3 \mathrm{Kev}$ to $28 \mathrm{Kev}$. Based on this observation, the discrete energies for simulated source $\mathrm{x}$-ray beam are chosen from $3 \mathrm{KeV}$ to $28 \mathrm{KeV}$, with an interval of $1 \mathrm{KeV}$.

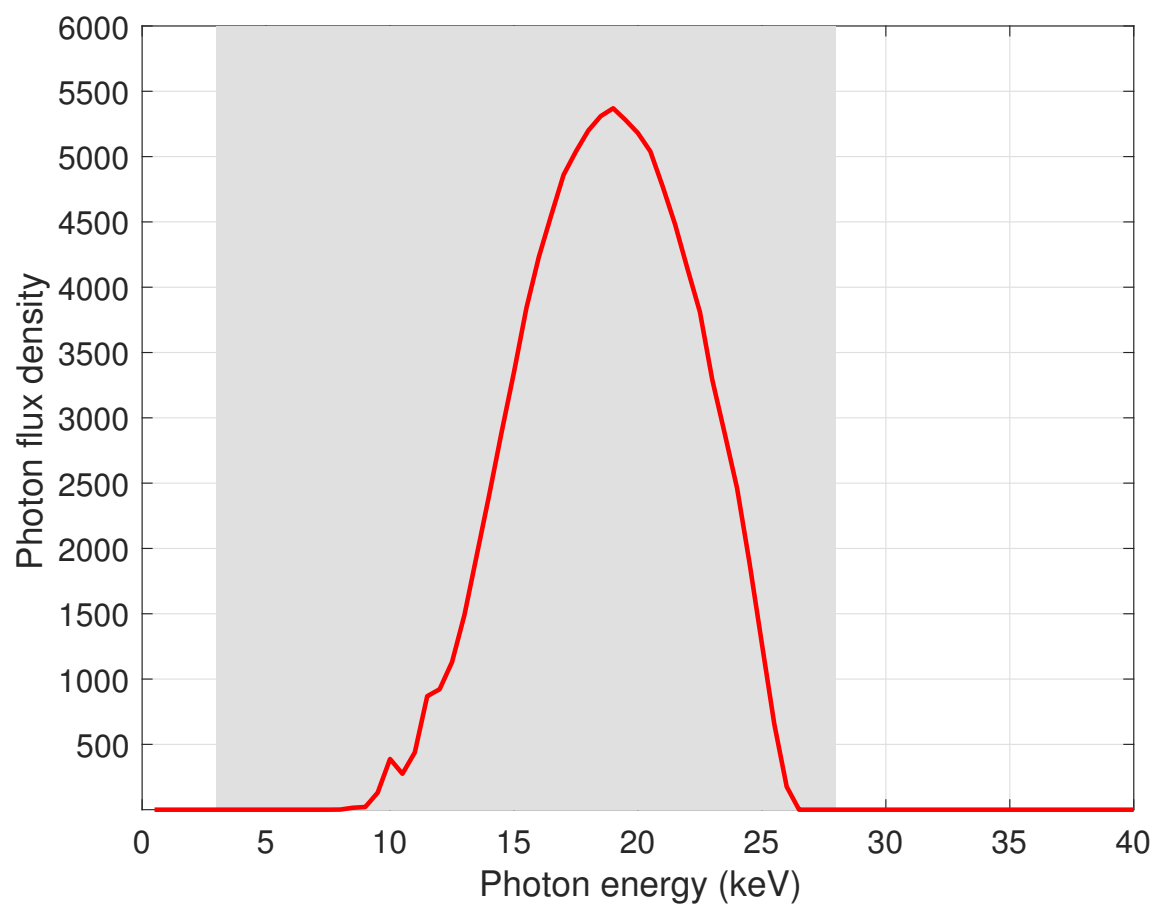

Figure 2. Photon flux density versus photon energy

The plots of linear attenuation coefficients to materials adipose, air and calcium are shown in Figure (3). In Figure (3), the red, blue and black curves represent adipose tissue, air and calcium, respectively, and the gray patch corresponds to the area of energy 


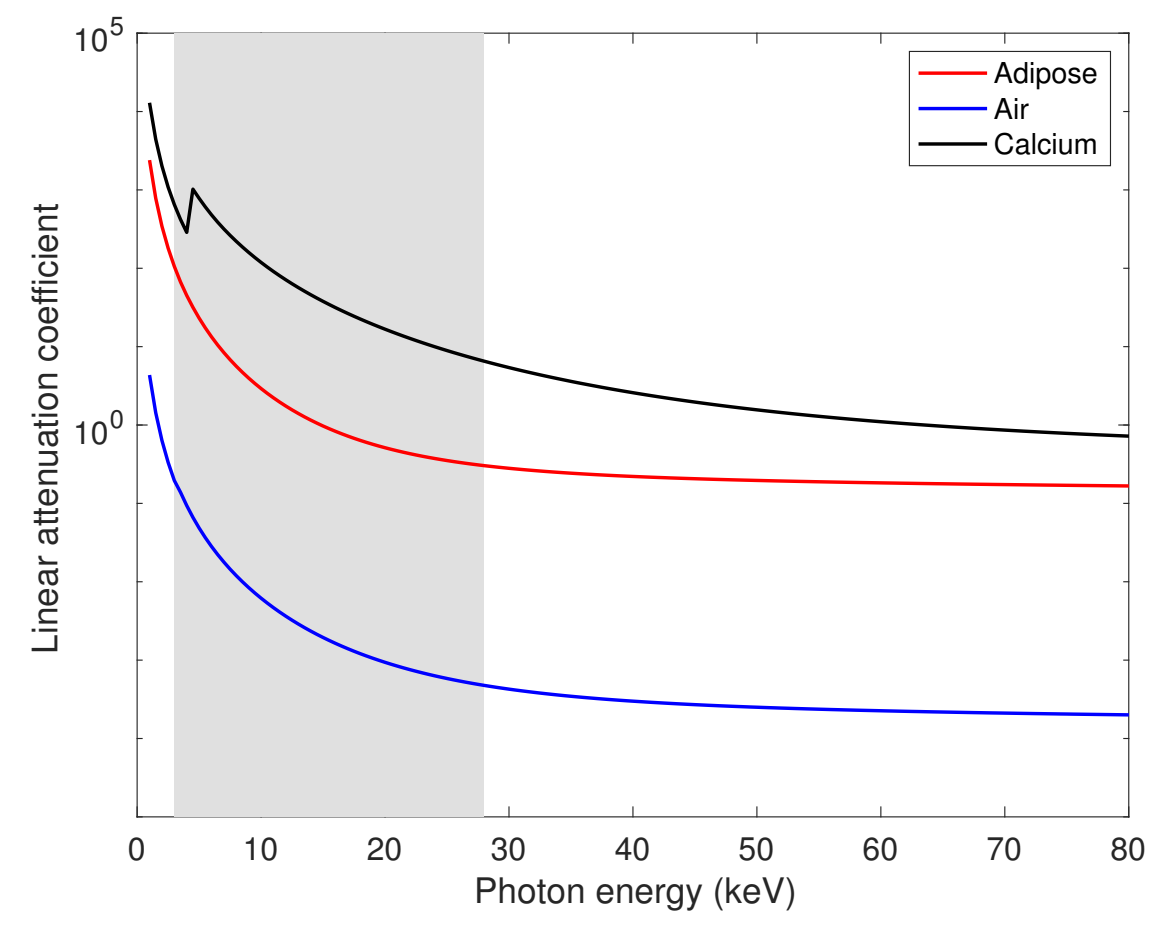

Figure 3. The linear attenuation curves for adipose (red), air (blue) and calcium (black).

flux that is not equivalent to zero. From Figure (3), we can see that the curvatures of air and adipose are similar, while the curve of calcium has a K-edge. The similarity of curvatures between adipose and air might cause collinearity of linear attenuation coefficient matrix $\boldsymbol{C}$ and so as ill-conditioning of Hessian, while the K-edge might result in difficulty of reconstruction.

The simulations of the true object, shown in Figure 4, contain four distinct regions: $100 \%$ adipose, $0 \%$ air, $0 \%$ calcium; $0 \%$ adipose, $100 \%$ air, $0 \%$ calcium; $0 \%$ adipose, $0 \%$ air, $100 \%$ calcium; $50 \%$ adipose, $50 \%$ air, $0 \%$ calcium $\ddagger$. The original images are available in Figure 4. Yellow color represents regions that contain 100\% of the corresponding material, the turquoise color indicates regions that contain $50 \%$ of the adipose and air materials, the blue color indicates that the material does not exist in this area. Since we only have three materials, then the weights corresponding to these three plots in Figure 4 should add to one. Moreover, since we are using a 2-dimensional object for the simulation, we use fan-beam (instead of cone beam) tomography model to generate a projection matrix $\boldsymbol{A}$. The distance between the source and detector is $70 \mathrm{~cm}$, with 2.5 $\mathrm{cm}$ air gap between the object and detector. In order to keep the gauge of projection matrix the same under different size of images, we scale the projection matrix by the grid size and the dimension of images. For example, we choose the grid size as $2 \mathrm{~cm}$

$\ddagger$ We actually tested many different combinations of mixed materials, for example, $20 \%$ adipose, $60 \%$ air and $20 \%$ calcium. The results are are very similar to the one case considered in this experiment, thus to conserve space, we omit the results. 

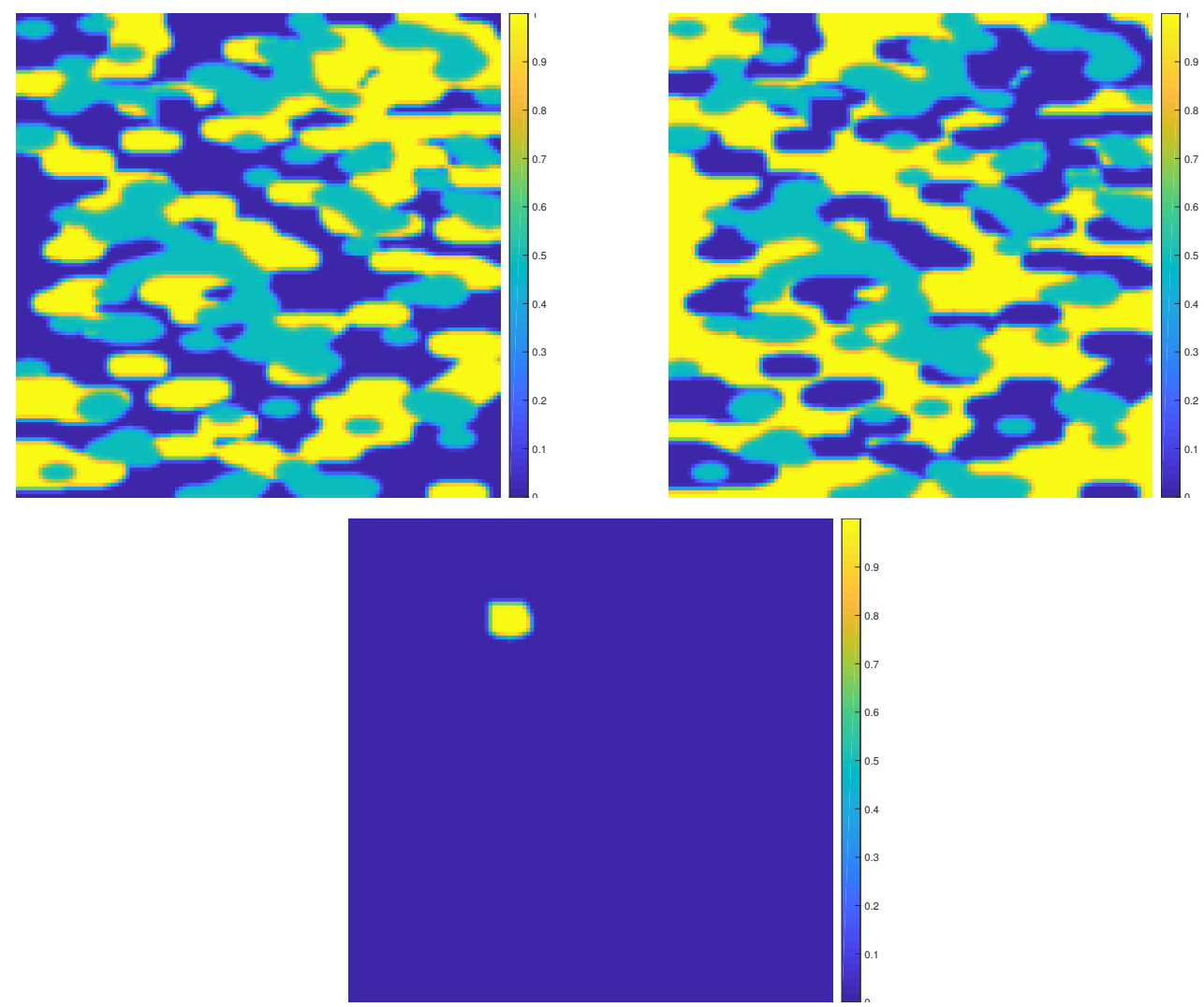

Figure 4. The true images for air (upper left), adipose (upper right) and calcium (middle). The turquoise colored regions are those areas in the object with a mixture of $50 \%$ glandular and $50 \%$ adipose tissue.

and the dimension as 128 pixels so the scaling results in a pixel size of 2/128 cm/pixel.

To avoid inverse crimes, we use spectral energies discretized on a finer grid and images with higher resolution to build the forward problem, but then use a coarser grid and lower resolution when solving the inverse problem. In particular, we collect the photon flux density corresponding to energies from $3 \mathrm{KeV}$ to $28 \mathrm{KeV}$, with an interval of $0.5 \mathrm{KeV}$ in the forward problem, but then use an interval of $1 \mathrm{KeV}$ when we solve the inverse problem. Moreover, the resolution of object is initially $256 \times 256$ when we build the forward problem, and for the inverse problem, we solve (using a function included in the package IR Tools [18]) on a $128 \times 128$ grid. The number of x-rays used in building ray trace matrix $\boldsymbol{A}$ is scaled to match the projected data generated with higher resolution images. Both full CT and limited angle reconstructions are presented in the following sections.

\subsection{Full Angle Reconstruction}

At first, we only consider the full CT case, where the range of projection angle is from 0 to 179 degrees in one degree increments. We use Poisson distribution to generate the measurements as (11). The initial guess is a random vector whose entries are between 0 and 1 and it is not required to satisfy the equality constraint. Moreover, 

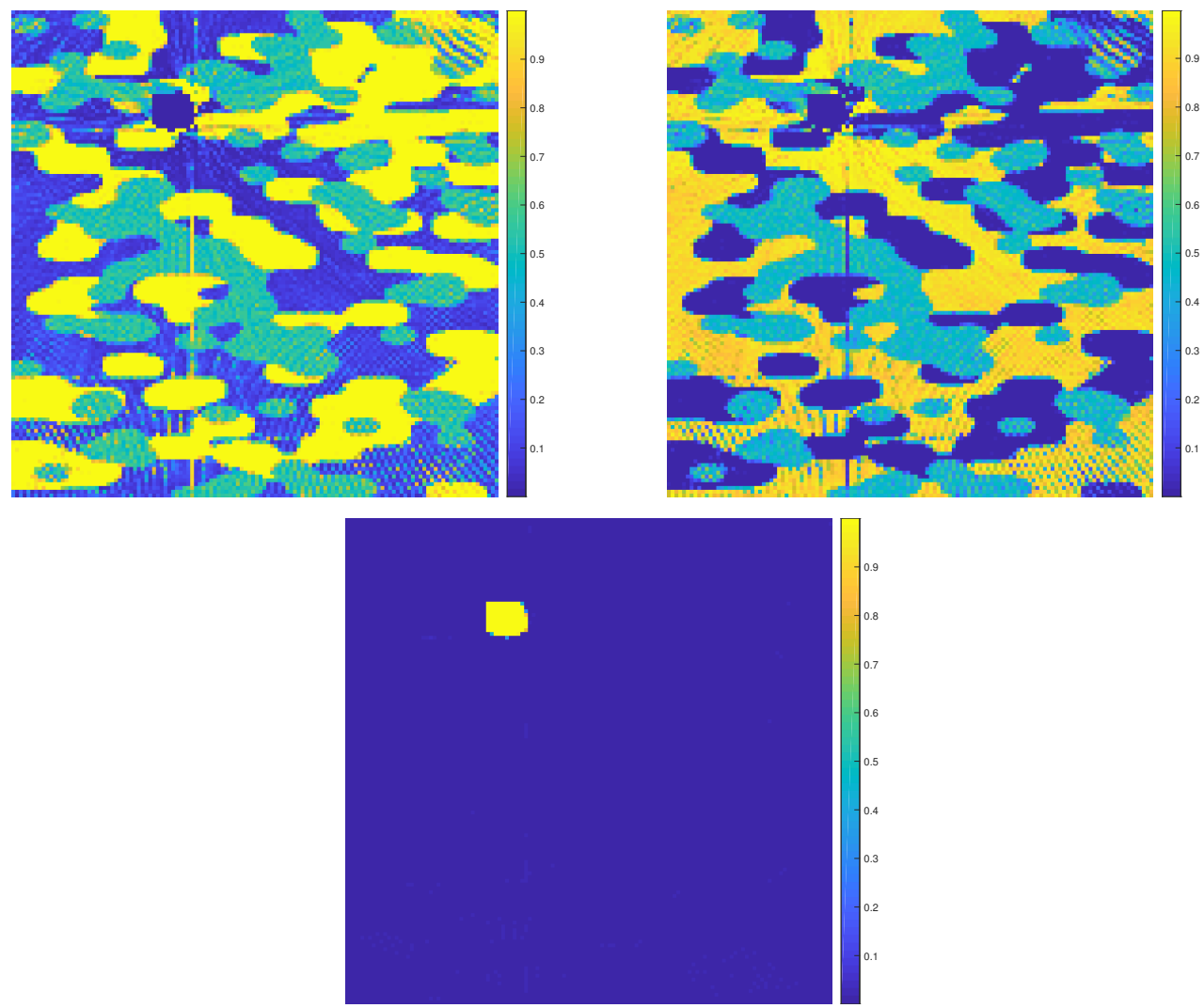

Figure 5. The reconstructed images for air (upper left), adipose (upper right) and calcium (middle) for full CT.

total variation regularization is introduced to preserve the edges; specifically, we use the central difference operator and zero boundary conditions for this regularization term. The regularization parameter is chosen among the set $\left\{10^{-1}, 10^{-2}, 10^{-3}, 10^{-4}, 10^{-5}\right\}$ and the most effective parameter is used to present the result. With our (un-optimized) MATLAB implementation on a laptop computer, we need around 20 minutes to finish 30 Newton iterations where the stationary point is achieved. For the full CT case, the reconstructed images are presented in Figure 5. From Figure 5, we can see that the reconstructed images are of high quality in general. It successfully separates the areas correponding to adipose, air and calcium as well as mixture of adipose and air. Edges of the reconstructed images are clear, which might be contributed from total variation regularization. On the other hand, we can also find several artifacts that appear as blurred spots concentrating in the upper right corner, as well as other small artifacts scattered around the image. This results from measured data that are generated by Poisson distribution. With lower radiation dose, the relative noise level is higher compared with higher dose. Moreover, we can illustrate the convergence behavior by investigating the curves of relative errors. This plot is shown in Figure 6.

From Figure 6, we can find that the relative errors of materials air and adipose decrease in a similar way while the relative error of material calcium drops much faster. It is likely that calcium is only composed of a small part of the area and it can achieve 


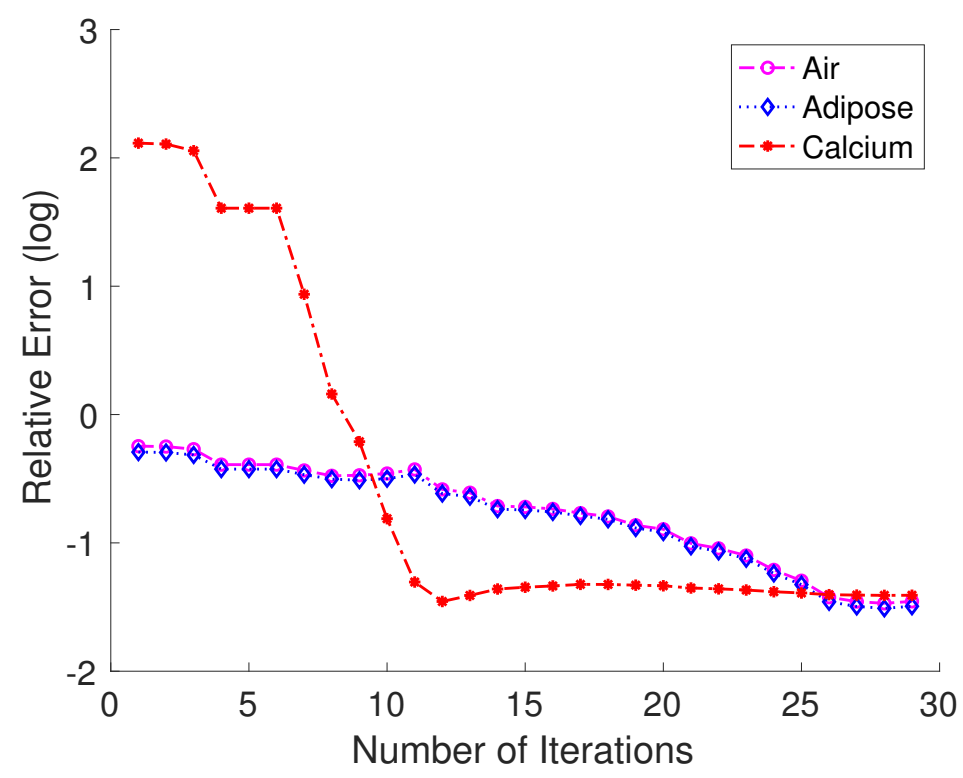

Figure 6. Plot of relative errors of air (magenta), adipose (blue) and calcium (red) for the full CT simulation.

faster convergence and higher accuracy. We also observe that the relative errors of three materials decrease to a particular level and then stagnate. The relative error between the last step and true solution is about $19 \%$ for each material. Note that this stagnation occurs because of the regularization - without regularization, the relative errors may actually increase as the iterations proceed, which is a well-known behavior of ill-posed inverse problems, referred to as semi-convergence. We observe that when the relative errors stagnate, the current step approaches the first order optimality condition, which is an approximate solution to the KKT system.

To further validate convergence behavior of the proposed algorithm, we plot the curve of function value in Figure (7). From Figure (7), we can clearly identify that the function value drops fast in the beginning and then it stops for two iterations. After that, it starts to drop again and then stagnate. It cannot achieve lower value when reaching a specific level.

\subsection{Limited Angle Reconstruction}

In addition to the full $\mathrm{CT}$ case, it is important to also consider the case of limited angle reconstructions. Specifically, in the area of digital tomography, the limited angle technique known as tomosynthesis has become an important diagnostic tool in breast imaging. The motivation for using limited angle techniques are to reduce radiation dose to patients as well as to reduce the cost of the procedure. The limited angle situation provides significantly more challenges to image reconstruction because the mathematical problems is much more ill-posed than the full CT case. This means that the reconstruction quality is much more sensitive to the noise. In addition, the original 


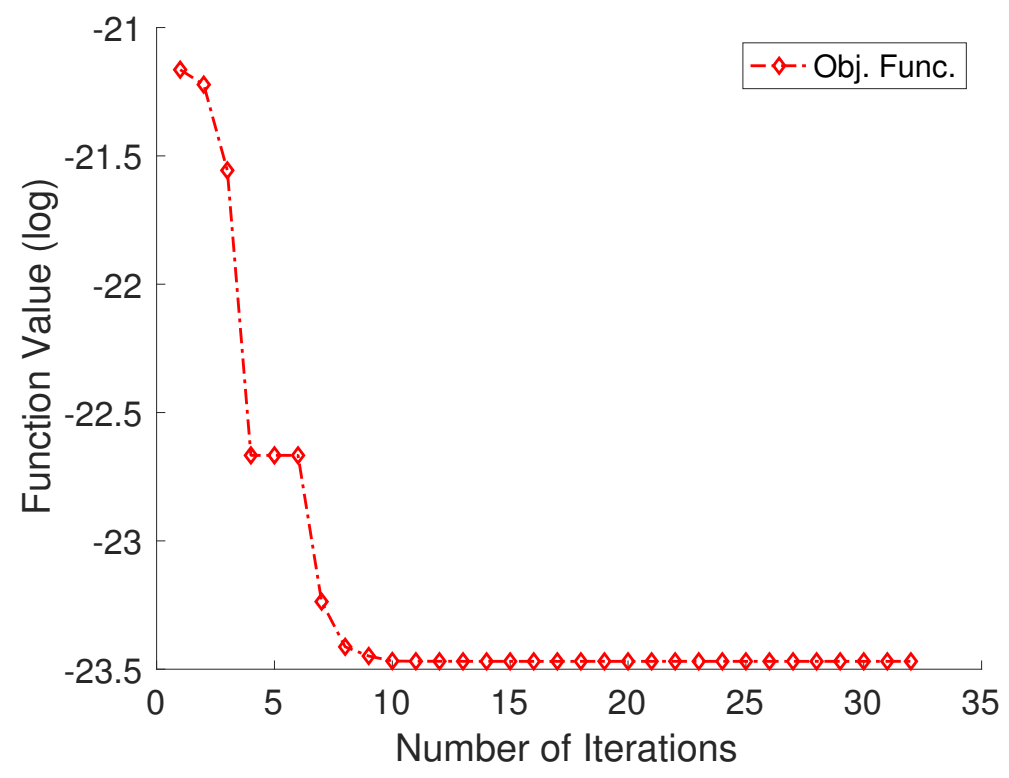

Figure 7. Plot of decrease of the objective function value

objective function might have more stationary points and several of them are likely to satisfy the KKT condition. Under this situation, when the problem is effectively underdetermined, a poor initial guess may lead to an undesirable local minimum.

To test the limited angle reconstruction, the same test problem is used but with fewer projection angles. We shrink the projection angles from 180 degrees to 90 degrees, which ranges from 0 to 90 degrees. Furthermore, Poisson distribution is used to generate the projection data. After implementing the previous algorithm, the reconstructed images are presented in Figure 8.

From Figure 8, we can clearly see that the reconstructed images generated from the limited angle case are more blurred than the images from the full CT case. As expected, with fewer projection angles, the images are of poorer quality. For the 90 degree case, we can basically identify the distributions of materials roughly, while the details are more difficult to recognize. Only the material map of calcium is nearly fully separated from other materials. Moreover, we can see that the boundaries of different materials are not as clear as in the full CT case. In several areas, the pixels are surrounded by shadows, which means that the materials are not completely separated. In the area of mixture, several pixels are colorful and the results depart slightly from the true solution. However, it is well known that due to the limited angle data, there are fundamental limitations when computing reconstructions [19].

The plot of relative errors for 90 degree case is presented in Figure 9. From Figure 9, we can find that the relative error plots corresponding to air and adipose decrease slowly and then stagnate. On the other hand, the relative error curve corresponding to calcium drops fast and converge to a lower level. This observation matches the phenomenon we conclude from the reconstructed images. Compared with full CT Figure 6, the relative 
Nonlinear Optimization for Mixed Attenuation Polyenergetic Image Reconstruction 21
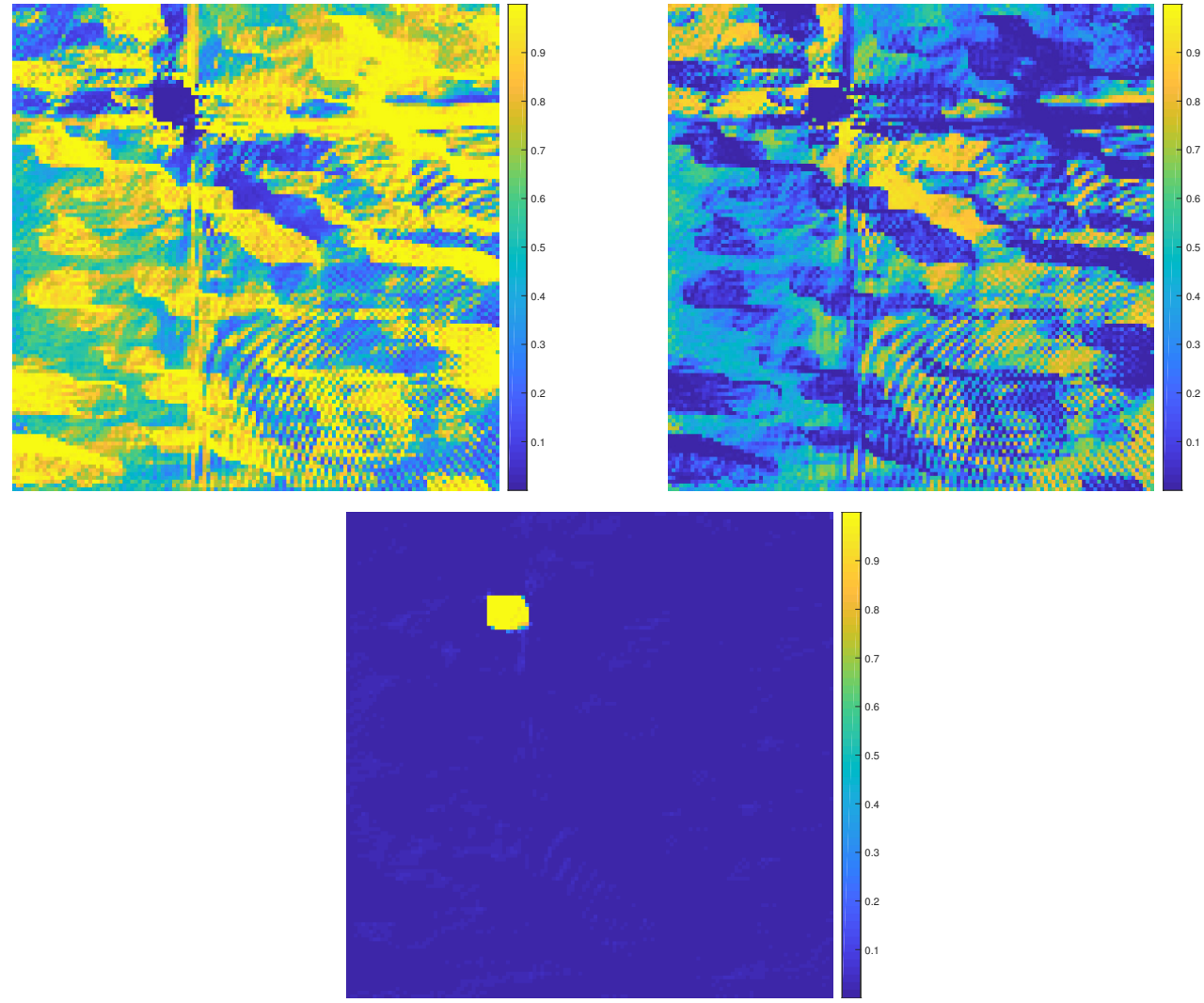

Figure 8. The reconstructed images for air (upper left), adipose (upper right) and calcium (middle) with 90 degree projection.

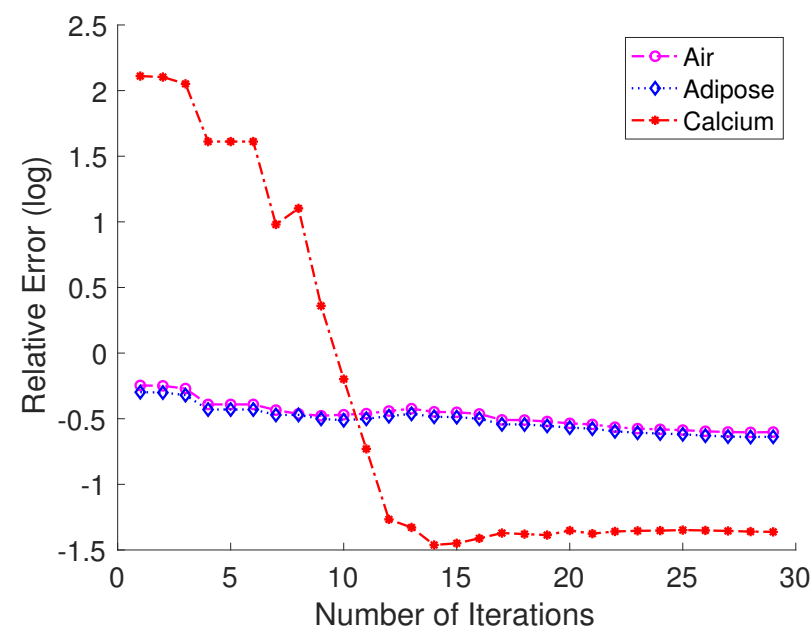

Figure 9. Plot of relative errors of air (magenta), adipose (blue) and calcium (red) for the 90 degree limited angle simulation.

errors corresponding to the flat steps are higher. Moreover, we can find that the speed of convergence is not as rapid as in the full CT case. However, we do observe that even if the regularization cannot completely compensate for the limited angles, it does help to stabilize the solution. 


\subsection{Comparison of Previous Work}

In the paper [5], Mejia-Bustamante et al. investigate a similar problem with only equality constraints. Under the assumption that projected data follow a Poisson distribution, they set up a likelihood function and obtain an optimization problem based on the negative log-likelihood, which is similar to the objective function used in this paper. Moreover, the use substitution to impose the equality constraints implicitly because the weights corresponding to each material can be represented by a linear combination of weights of other materials. On the other hand, Chung et al. [4] compare the performance of different optimization methods, such as gradient descent and Newton-CG under similar setup. Since we already include Newton-CG method in the trust region subproblem, we can compare gradient descent method with the method we use. To further impose the bound constraints, we add a line search scheme to the gradient descent method. We generate an object of only two materials, adipose and calcium, and they compensate each other. Using the method mentioned in [5], we can use the weights of calcium to represent the weights of adipose since they add to one. The original images are similar to the first two images in Figure (4). The statistics are collected in Table 1 and Table 2.

\begin{tabular}{ccccc}
\hline \multicolumn{5}{c}{ Projected Gradient Descent } \\
\hline Iteration & Rel. Grad. & Num. Fun. Eval. & Rel. Err. 1st & Rel. Err. 2nd \\
\hline 1 & 1.00 & 19 & 0.7566 & 0.7291 \\
5 & 0.6084 & 115 & 0.7389 & 0.7119 \\
10 & 0.1502 & 209 & 0.7282 & 0.7016 \\
25 & 0.0703 & 491 & 0.7079 & 0.6821 \\
50 & 0.0416 & 943 & 0.6883 & 0.6632 \\
\hline
\end{tabular}

Table 1. Statistics for projected gradient descent method

\begin{tabular}{cccccc}
\hline \multicolumn{5}{c}{ Nonlinear Interior Point Trust Region Method } \\
\hline Iteration & Rel. Grad. & Num. Fun. Eval & Rel. Err. 1st & Rel. Err. 2nd & CG Iter. \\
\hline 1 & 1.00 & 7 & 0.8022 & 0.7672 & 1 \\
5 & 0.8052 & 49 & 0.6409 & 0.6179 & 2 \\
10 & 0.1123 & 139 & 0.5499 & 0.5298 & 28 \\
15 & 0.0117 & 852 & 0.3699 & 0.3564 & 264 \\
20 & 0.0050 & 3752 & 0.3166 & 0.3051 & 372 \\
\hline
\end{tabular}

Table 2. Statistics for nonlinear interior point trust region method

From Table 1 and Table 2, we can clearly observe that the nonlinear interior point trust region method can achieve much faster convergence than the projected gradient descent method under similar costs. For example, with 50 iterations and 943 function 
evaluations, the projected gradient descent method can reduce the relative error to around 68\%. However, with 15 Newton iterations and 852 function evaluations, the relative error can be reduced to approximate $37 \%$ by the nonlinear interior point trust region method.

\section{Conclusions and Remarks}

By taking multiple materials into consideration, the reconstructed images can reveal the weights of materials that compose this object, providing substantially more useful information to the clinician. Furthermore, the objective function and gradient are uncomplicated to implement and the modified Hessian is a sufficient and stable estimate to the true Hessian. In addition, the merits of using a nonlinear interior-point method are easy to identify. It is a globally convergent method with superlinear rate of convergence. It is also a stable and robust algorithm that can handle large-scale problems. Furthermore, there is substantial flexibility in choosing the initial guess because it does not need to satisfy the constraints. Moreover, as was illustrated in our numerical experiments, we can speed up the algorithm with the proposed scaling method.

Although it has many advantages, it still has several limitations. For example, implementation of nonlinear interior-point method is not straightforward for large-scale problems. It requires solving a normal subproblem as well as a tangential subproblem. Furthermore, we need to decide the size of trust region in each iteration. Meanwhile, this method involves many parameters that we need to choose manually. So far, we have only tested 2D images rather than 3D images. For 3D images, the evaluation of each part might be more complicated, which is likely to increase the expense of solving this problem. For further research, we might consider the gradient-based methods such as the scaled gradient descent method or splitting methods such as the alternating direction method of multipliers (ADMM) [20].

\section{Acknowledgment}

This work is supported by the US National Science Foundation under grant no. DMS1819042. The content is solely the responsibility of the authors and does not necessarily represent the official views of these institutions.

\section{References}

[1] J Peter Stonestrom, Robert E Alvarez, and Albert Macovski. A framework for spectral artifact corrections in X-ray CT. IEEE Transactions on Biomedical Engineering, (2):128-141, 1981.

[2] Xiao Zhu Lin, Fei Miao, Jian Ying Li, Hai Peng Dong, Yun Shen, and Ke Min Chen. High-definition CT Gemstone spectral imaging of the brain: initial results of selecting optimal monochromatic 
image for beam-hardening artifacts and image noise reduction. Journal of Computer Assisted Tomography, 35(2):294-297, 2011.

[3] Idris A Elbakri and Jeffrey A Fessler. Statistical image reconstruction for polyenergetic x-ray computed tomography. IEEE transactions on medical imaging, 21(2):89-99, 2002.

[4] Julianne Chung, James G Nagy, and Ioannis Sechopoulos. Numerical algorithms for polyenergetic digital breast tomosynthesis reconstruction. SIAM Journal on Imaging Sciences, 3(1):133-152, 2010 .

[5] Veronica Mejia Bustamante, James G Nagy, Steve SJ Feng, and Ioannis Sechopoulos. Iterative breast tomosynthesis image reconstruction. SIAM Journal on Scientific Computing, 35(5):S192S208, 2013.

[6] V. M. Bustamante. Iterative Polyenergetic Digital Tomosynthesis Reconstructions for Breast Cancer Screening. PhD thesis, Emory University, 2013.

https://etd.library.emory.edu/file/view/pid/emory:d6wn4/mejia\% 20bustamante_dissertation.pdf.

[7] Rina Foygel Barber, Emil Y Sidky, Taly Gilat Schmidt, and Xiaochuan Pan. An algorithm for constrained one-step inversion of spectral CT data. Physics in Medicine and Biology, 61(10):3784, 2016.

[8] Charles L Epstein. Introduction to the mathematics of medical imaging. SIAM, 2007.

[9] Xiaochuan Pan, Emil Y Sidky, and Michael Vannier. Why do commercial CT scanners still employ traditional, filtered back-projection for image reconstruction? Inverse problems, 25(12):123009, 2009.

[10] Jennifer L Mueller and Samuli Siltanen. Linear and nonlinear inverse problems with practical applications. SIAM, 2012.

[11] Björn J Heismann, Bernhard T Schmidt, and Thomas Flohr. Spectral computed tomography. SPIE Bellingham, WA, 2012.

[12] Per Christian Hansen, James G Nagy, and Dianne P O'leary. Deblurring Images: Matrices, Spectra, and Filtering, volume 3. SIAM, 2006.

[13] Jorge Nocedal and Stephen J Wright. Sequential quadratic programming. Springer, 2006.

[14] Richard Byrd, Jorge Nocedal, and Richard Waltz. K nitro: An integrated package for nonlinear optimization. Large-scale nonlinear optimization, pages 35-59, 2006.

[15] Richard H Byrd, Mary E Hribar, and Jorge Nocedal. An interior point algorithm for large-scale nonlinear programming. SIAM Journal on Optimization, 9(4):877-900, 1999.

[16] Trond Steihaug. The conjugate gradient method and trust regions in large scale optimization. SIAM Journal on Numerical Analysis, 20(3):626-637, 1983.

[17] JH Siewerdsen, AM Waese, DJ Moseley, S Richard, and DA Jaffray. Spektr: A computational tool for x-ray spectral analysis and imaging system optimization. Medical physics, 31(11):3057-3067, 2004.

[18] Silvia Gazzola, Per Christian Hansen, and James G Nagy. IR Tools: A MATLAB package of iterative regularization methods and large-scale test problems. Numer. Algorithms, https://doi.org/10.1007/s11075-018-0570-7, 2018.

[19] Jürgen Frikel and Eric Todd Quinto. Characterization and reduction of artifacts in limited angle tomography. Inverse Problems, 29(12):125007, 2013.

[20] Daniel Gabay and Bertrand Mercier. A dual algorithm for the solution of nonlinear variational problems via finite element approximation. Computers \&3 Mathematics with Applications, 2(1):17-40, 1976.

[21] Roy Mathias. The spectral norm of a nonnegative matrix. Linear Algebra and its Applications, 139:269-284, 1990.

[22] Rina Foygel Barber and Emil Y Sidky. Mocca: mirrored convex/concave optimization for nonconvex composite functions. Journal of Machine Learning Research, 17(144):1-51, 2016.

[23] Julianne M Chung, Misha E Kilmer, and Dianne P O'Leary. A framework for regularization via operator approximation. SIAM Journal on Scientific Computing, 37(2):B332-B359, 2015. 
[24] Stephen G Nash. Preconditioning of truncated-newton methods. SIAM Journal on Scientific and Statistical Computing, 6(3):599-616, 1985.

[25] AS El-Bakry, Richard A Tapia, T Tsuchiya, and Yin Zhang. On the formulation and theory of the newton interior-point method for nonlinear programming. Journal of Optimization theory and Applications, 89(3):507-541, 1996.

[26] Joonki Noh, Jeffrey A Fessler, and Paul E Kinahan. Statistical sinogram restoration in dual-energy CT for PET attenuation correction. IEEE Transactions on Medical Imaging, 28(11):1688-1702, 2009.

[27] Richard H Byrd, Jean Charles Gilbert, and Jorge Nocedal. A trust region method based on interior point techniques for nonlinear programming. Mathematical Programming, 89(1):149-185, 2000.

[28] Nargol Rezvani. Iterative reconstruction algorithms for polyenergetic $x$-ray computerized tomography. PhD thesis, University of Toronto, 2012.

[29] Patrick L Combettes and J-C Pesquet. Image restoration subject to a total variation constraint. IEEE Transactions on Image Processing, 13(9):1213-1222, 2004.

[30] Alexander H Delaney and Yoram Bresler. Globally convergent edge-preserving regularized reconstruction: an application to limited-angle tomography. IEEE Transactions on Image Processing, 7(2):204-221, 1998.

[31] Curtis R Vogel. Computational methods for inverse problems. SIAM, 2002.

[32] Jan Modersitzki. FAIR: flexible algorithms for image registration. SIAM, 2009.

[33] Silvia Bonettini, Alessandro Chiuso, and Marco Prato. A scaled gradient projection method for bayesian learning in dynamical systems. SIAM Journal on Scientific Computing, 37(3):A1297A1318, 2015.

[34] JB Francisco, N Krejić, and JM Martínez. An interior-point method for solving box-constrained underdetermined nonlinear systems. Journal of Computational and Applied Mathematics, 177(1):67-88, 2005.

[35] Gabor T Herman and Ran Davidi. Image reconstruction from a small number of projections. Inverse problems, 24(4):045011, 2008.

[36] Gene H Golub, Per Christian Hansen, and Dianne P O'Leary. Tikhonov regularization and total least squares. SIAM Journal on Matrix Analysis and Applications, 21(1):185-194, 1999.

[37] Per Christian Hansen and Maria Saxild-Hansen. AIR Tools: A MATLAB package of algebraic iterative reconstruction methods. Journal of Computational and Applied Mathematics, 236(8):2167-2178, 2012.

[38] Kwang Eun Jang, Jongha Lee, Younghun Sung, and SeongDeok Lee. Information-theoretic discrepancy based iterative reconstructions (idir) for polychromatic x-ray tomography. Medical physics, 40(9), 2013.

[39] Jonathan H Mason, Alessandro Perelli, William H Nailon, and Mike E Davies. Polyquant CT: direct electron and mass density reconstruction from a single polyenergetic source. Physics in Medicine \&3 Biology, 62(22):8739, 2017.

[40] Thomas Blumensath and Richard Boardman. Non-convexly constrained image reconstruction from nonlinear tomographic x-ray measurements. Phil. Trans. R. Soc. A, 373(2043):20140393, 2015 . 